\title{
On consumable energy allocation frame-work with SWIPT for remotely located wireless node within internet of things
}

\author{
AlaaAllah Ahmed ElSabaa*, Mohammad Patwary ${ }^{\dagger}$, Elhadj Benkhelifa ${ }^{\ddagger}$, Amira Zaki* and Said E. El-Khamy ${ }^{\S}$ \\ *Electronics and Communications Dept., Arab Academy for Science and Technology, Alexandria, Egypt. \\ ${ }^{\dagger}$ School of Computing and Digital Technologies, Birmingham City University, Birmingham, UK. \\ $\ddagger$ School of Computing and Digital Technology, Staffordshire University. \\ $\S$ Department of Electrical Engineering, Faculty of Engineering Alexandria University, Alexandria, Egypt.
}

Emails: elsab3.92@gmail.com, mohammad.patwary@bcu.ac.uk, E.Benkhelifa@ staffs.ac.uk, amzak10@aast.edu, elkhamy@ieee.org

\begin{abstract}
The degree of assurance of providing reliable connectivity to diversely located devices will define the success of internet of things as aimed for future generation of wireless networks. This poses a challenge of making energy available to such devices, regardless of the environment or the circumstances they are into to keep them connected. Solving such a challenge is expected to provide significant degree of flexibility to the coverage planning of the future generations of wireless networks. Simultaneous wireless information and power transfer (SWIPT) provides a candidate solution to resolve this problem; which may require a trade off with the available data rate to the device. This paper proposes an innovative trade-off frame-work to avoid any of the data rate compromise, while providing enormous flexibility to facilitate energy to the remotely located devices on demand basis. Such flexibility relaxes the coverage as well as capacity planning constraint in future wireless networks.
\end{abstract} ing.

Index Terms-SWIPT, Internet of Things, Compressive Sens-

\section{INTRODUCTION}

Energy constrained components within modern communication systems operating with traditional power supply such as; remotely located relays or end users; suffer from operational lifetime deterioration. To sustain network connectivity and continuity, batteries are regularly required to be recharged or replaced. This constraint is nevertheless costly and inconvenient within the nature of applications. Consequently, exploring various solutions to this drawback is crucial. Emerging solutions included energy harvesting, that is scavenging energy from external natural resources such as solar or wind etc. However, the amount of energy harvested from such natural resources is intermittent in nature, as they depend on some uncontrollable factors such as the weather conditions, which makes reliable communication difficult.

An appealing approach that overcomes the reliability concern is to harvest energy from directive electromagnetic radiation of radio frequency (RF), which is also known as wireless power transfer (WPT). The compelling fact of RF signals can transfer both information and energy brought tremendous attraction, the technique that is widely accepted as simultaneous wireless information and power transfer (SWIPT).
Performance tradeoffs between information and power transmission within SWIPT were studied over flat fading channels in [1] and frequency selective channels in [2], both under the assumption of simultaneous energy and information decoding of the same received signal by the receiver. However, practical circuits for energy harvesting $(\mathrm{EH})$ from radio signals are not yet capable of directly decoding the carried information. Consequently, practical circuit designs, namely time switching (TS) and power switching (PS) were proposed in [1]. As found in Fig.1, and throughout the context, we will be denoting base station,relay and end-user as S,R and EU, respectively.

- Scenario 1: Demonstrates the common case where the S-EU link is reliable, i.e the end user falls under the network coverage area, yet runs out of energy to communicate back and so $\mathrm{S}$ can either exchange data with the EU or consider simultaneously transmitting data and empowering it with energy to aid its operation during the UL.

- Scenario 2: takes place when EU are remote with only sufficient energy to communicate to a shorter distance and thus the relay's assistance is a necessity for the information exchange between the source and end users. In return, $S$ supplies both information to the $R$ for processing then forwarding to the EU and enough energy to supply its full operations.

Employing relays to enhance the coverage as well as capacity of a SWIPT dependent system has drawn significant attention. As presented in [3], where authors assumed both the source and the relay had conventional power supply and the end users has energy harvesting $(\mathrm{EH})$ capabilities as their work focused on achieving different tradeoffs for SWIPT by optimizing system parameters; such as transmit power and power splitting factors. In [4], SWIPT for a decode-and- forward (DF) relay systems where the relays are considered to be self sustained with their own energy harvesting capabilities. Additionally, full-duplex energy constrained relaying system was examined in [5] to derive an analytical characterization of the achievable 
throughput of three different communication modes and their equivalent optimum time split mode.

Considering, the fact that many natural signals possess an inherent characteristic, sparsity, that enables the signal to be stored in few samples and subsequently be recovered accurately, courtesy of compressive sensing (CS). Statistically, most of the remotely located devices are expected to be sensor node, whose responses are sparse in nature. Contrary to traditional Nyquist paradigm, the CS paradigm, banking on finding sparse solutions to under determined linear systems, can reconstruct the signals from far fewer samples than is possible using Nyquist sampling rate [6],[7]. In [8] performance parameters of CS techniques had been studied for various reconstruction algorithms for comparison.

As next generation networks are expected to have higher connectivity and improved coverage, suggestions for reaching out to unapproachable end users, are being studied intensively. Since secondary aiding elements (relay) employment already proved improvement in the system capacity and communication coverage. Therefore, we suggest the presence of EH DF relays to assist the source-to-enduser communication link.

The different multiplexing schemes and multiple access techniques that can be considered during the UL and DL of the different SWIPT schemes employing relays along with the direction of information transfer (IT) and $\mathrm{EH}$ for each scenario separately. For simplicity, we have chosen to consider that during the $\mathrm{DL}$ from the $\mathrm{S}$ to the $\mathrm{R}$ and during the $\mathrm{UL}$ from the EU to the R, information and energy are both separated over time i.e time division multiple access (TDMA) is adopted. On the other hand, space division multiple access (SDMA) is used in the DL from the $\mathrm{R}$ to the EU, hence $\mathrm{R}$ transceivers beam-forming is demanded, as we presume the network to operate in FDD mode i.e. two different channels are used for UL and DL simultaneously.

Within the scope of authors' knowledge, none of the previous works considered studying system throughput and energy efficiency using data compression (compressive sensing) after optimizing the time and power allocation of $\mathrm{EH}$ and IT. Motivated by the fact that, the extra processing we are proposing demands trivial amount of energy when compared to the energy required to transmit more chunks of data. This work aims to achieve the maximum system throughput while maintaining the end users grade of service as well as data rate. This work considers relay-aided low power wireless communication system, where the relays operate using EH. In such scheme,the following problem has been addressed:

- The use of relays in cases where the actual network coverage is beneath the anticipated design.

- Various schemes for EH by relays depending on the energy required to fulfil different tasks.

- Establishing a framework for performance tradeoffs by applying compressing sampling techniques to attain a constant data rate.

The main contributions of this paper are listed as follows: 1) Providing a framework by jointly optimizing time and power allocation trade-offs, such that the harvested energy is maximized, while guaranteeing the transmission rate achieving the target rate.

2) The proposed framework also indented to use compressive sensing to attain fixed data rate while allocating variable time slot fractions for energy harvesting in response to the nature of demand. The remainder of this paper is organized as follows. In section II, the system model is introduced and the problem formulation and the proposed optimal solution are derived in section III . Numerical results and discussion are shown in section IV and finally the conclusion and future work are presented in section V.

\section{System Model AND PROBlem Formulation}

For simplified realization of the scope of the problem, this paper considers a wireless system with single base station $S$ required for the area coverage of our interest, a relay $R$ and multiple end users $D_{j}$ are employed. Each end user D employs only one transceiver while the $\mathrm{BS} S$ and the $R$ is equipped with dual antennas (one antenna for transmission and the other for reception) for full duplex operation. Here, the $\mathrm{S}$ is assumed to be powered by a fixed source, which is not energy constrained. Throughout this paper, we assume that all the channel matrices are perfectly known to all nodes. The channel between $S$ and $R$, and $R$ and destination $D_{j}$ is denoted by $\mathbf{H}_{k, i}$ and $\mathbf{H}_{j, k}$, respectively. While the channel between each transmitting source antenna and receiving relay antenna and the channel between each relay transmitting antenna and the end user's antenna are modelled as $\mathbf{h}_{r, s}$ and $\mathbf{h}_{d, r}$,respectively.

In this section, the optimization problem regarding time and power allocation such that the energy harvested is maximized while guaranteeing the transmission rate constraint is formulated. Along with, considering the capacity of FD relaying channel,compressive sensing performance boundaries is defined as a final benchmark.

1) Optimizing time and power:

The received signal over one time slot at the relay is divided into two sub-slots, with duration of $\alpha \mathrm{T}$ and (1$\alpha) \mathrm{T}$, where $\alpha \mathrm{T}+(1-\alpha) \mathrm{T}=\mathrm{T}$. The factor $\alpha$ range between $[0,1]$, as $\alpha$ is expected to be governed by the dynamic energy demand variation w.r.t environment or circumstantial changes of the devices; in the context of its consumable energy availability. As shown in Fig.2, the frame $\alpha$ T represents the time taken in energy harvesting and (1- $\alpha) \mathrm{T}$ is the frame used to transfer information, by the relay from the source. The achievable rate for the $\mathrm{S}-\mathrm{R}$ link is given by;

$$
R_{S}=(1-\alpha) T \log \left(1+\frac{\left|\mathbf{h}_{k, i}\right|^{2} P_{S I}}{\sigma_{r}^{2}}\right)
$$

While the harvested energy,for the same link, can be written as;

$$
E_{E H}=\alpha T \eta\left(P_{S E}\left|\mathbf{h}_{k, i}\right|^{2}\right)
$$






Fig. 1. Scenarios considered employing relay aided SWIPT systems variously
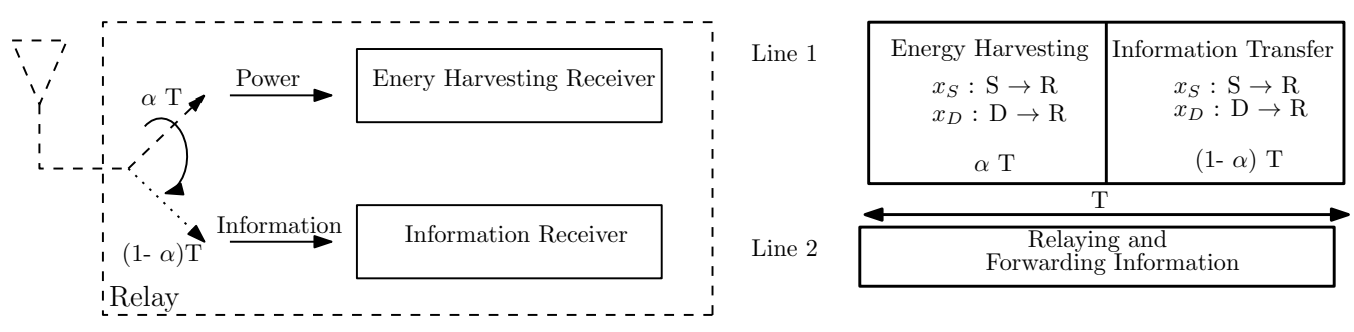

Fig. 2. Time switching relay operational sequence consideredin the proposed framework

With the objective of maximizing the harvested energy by joint power and time allocation subjected to a given target transmission rate constraint, the following optimization problem is formulated.

$$
\begin{array}{ll}
\underset{E_{E H}}{\operatorname{maximize}} & \alpha T \eta\left(P_{S E}\left|\mathbf{h}_{k, i}\right|^{2}\right) \\
\text { subject to } & (1-\alpha) T \log \left(1+\frac{\left|\mathbf{h}_{k, i}\right|^{2} P_{S I}}{\sigma_{r}^{2}}\right) \succeq R_{T} \\
& \alpha T P_{S E}+(1-\alpha) T P_{S I} \preceq T P_{S} .
\end{array}
$$

The joint optimization of power allocation $\mathbf{P}=$ $\left\{P_{S I}, P_{S E}\right\}$ and time splitting fractions $\mathbf{T}=\{\alpha T,(1-$ $\alpha) T\}$ to maximize the energy harvested $E_{E H}$ while guaranteeing the transmission rate achieving the target rate $R_{T}$ can be formulated as dual function optimization problem which is solved with Lagrangian dual function solution, denoted by $g(\beta)$ where;

$$
g(\beta)=\max _{P, T} L(P, T)
$$

where $\beta=\left(\beta_{1}, \beta_{2}\right)$ is the dual variables vector subject to the power and time constraints. Then, the dual optimization problem can be written as:

$$
\begin{array}{ll}
\min _{\beta} & \mathrm{g}(\beta) \\
\text { subject to } & \beta \succeq 0
\end{array}
$$

Since a dual function can be proved to be convex [], the subgradient-based method can be used to minimize $g(\beta)$ with guaranteed convergence. The subgradient can be easily given as

$$
\begin{gathered}
\Delta \beta_{1}=\alpha T \log \left(1+\frac{\left|\mathbf{h}_{k, i}\right|^{2} P_{S I}}{\sigma_{r}^{2}}\right)-R_{T} \\
\Delta \beta_{2}=T P_{S}-\alpha T P_{S E}+(1-\alpha) T P_{S I}
\end{gathered}
$$

Letting $\Delta \beta=\left(\Delta \beta_{1}, \Delta \beta_{2}\right), \beta$ is updated as $\beta^{t+1}=\beta^{t}+$ $\nu^{t} \Delta \beta$. With the step size $\nu^{t}$ following the diminishing step size policy, this subgradient method is guaranteed to converge to the optimal $\beta$.

Optimizing $\mathrm{P}$ and $\mathrm{T}$ with given dual variables. Calculating the Lagrange dual function $g(\beta)$ needs to obtain the optimal $\mathrm{P}$ and $\mathrm{T}$ at a given $\beta$, which can be executed with the following two steps.

A) Step 1- Obtaining the optimal $P$ for a fixed $T$ :

For a fixed $\mathrm{T}$ the partial derivatives of the Lagrangian in (23) with respective to the optimization variables $P_{S I}, P_{S E}$ are given by

$$
\frac{\partial L(P, T)}{\partial P_{S E}}=\alpha T\left(\eta\left|\mathbf{h}_{k, i}\right|^{2}-\beta_{2}\right)
$$

$$
\frac{\partial L(P, T)}{\partial P_{S I}}=(1-\alpha) T\left[\frac{\beta_{1}\left|\mathbf{h}_{k, i}\right|^{2}}{\sigma^{2}+\left|\mathbf{h}_{k, i}\right|^{2} P_{S I}}-\beta_{2}\right]
$$

By the Karush-Kuhn-Tucker conditions, the partial derivative of the Lagrangian is equal to zero at the 
optimal solution. Hence, the optimal $P_{S I}$ for a given $\beta$ can be obtained as

$$
P_{S I}^{*}=\frac{\beta_{2}}{\beta_{1}(1-\alpha) T}-\frac{\sigma^{2}}{\left|\mathbf{h}_{k, i}\right|^{2}}
$$

And the optimal $P_{S E}$ can be obtained as;

$$
P_{S E}^{*}= \begin{cases}P_{\max }, & T \alpha \eta\left|\mathbf{h}_{k, i}\right|^{2}>\beta_{2} . \\ P_{\min }, & T(1-\alpha) \eta\left|\mathbf{h}_{k, i}\right|^{2} \leq \beta_{2} .\end{cases}
$$

where $P_{\min }$ and $P_{\max }$ denote the minimum and maximum power constraints of the energy harvested by the relay.

B) Step 2 - Obtaining the optimal T:

Substituting (10) and (11) into (23), and adopting algebraic transformation, we can rewrite the Lagrangian in (23) as (24).

Where

$F_{I}^{*}=(1-\alpha) T\left[\beta_{1} \log \left(1+\frac{\left|\mathbf{h}_{j, k}\right|^{2} P_{S I}^{*}}{\sigma^{2}}\right)-\eta\left(P_{S I}^{*}\left|\mathbf{h}_{j, k}\right|^{2}\right)\right]$

In (24), it can be found that the only part that involves $(1-\alpha) T$ is $F_{I}^{*}$ and thus, the optimal $[(1-\alpha) T]^{*}$ can be found by;

$$
[(1-\alpha) T]^{*}=\max _{(1-\alpha) T} F_{I}^{*}
$$

That follows that the optimal $[\alpha T]^{*}$ can be given by;

$$
[\alpha T]^{*}=T-[(1-\alpha) T]^{*}
$$

2) Compressive Sensing Performance Bound:

By applying CS to chunks of data at the $\mathrm{S}$ node then decompressing them upon their arrival to the EU, there rises an interest in knowing how many chunks of data can be compressed while maintaining the probability of error threshold. Considering the work done by [9], were they analysed the performance of noisy compressive sensing theoretically, and derived the upper and lower bound of the compressive sensing probability of error. For the $\mathrm{N}$-dimensional signal vector $\mathrm{x}$ which is k-sparse in some domain $(\Psi)$, and can be characterised by a less number of measurements $\mathrm{M}$, such that $\mathrm{M} \ll \mathrm{N}$, and referring to $\mathrm{M} / \mathrm{N}$ as the sampling rate, with the assumptions of;

a) Both the original information and noise follow the Gaussian distribution.

b) the source information $x \in \mathbb{R}^{\mathbb{N}}$ is $\mathrm{K}$ - sparse in the basis $\Psi$, and each of them is i.i.d. Gaussian random variable with zero mean and variance $\sigma_{x}^{2}$.

c) The measurement matrix $\Phi$ is Bernoulli matrix, that is, the entries of $\phi_{m, n}$ are i.i.d with $\operatorname{Pr}\left(\phi_{m, n}= \pm 1\right)$ $=0.5$. In addition, the measurement matrix $\Phi$ is known for both the transmitter and the receiver in advance.

d) Each element of the noise vector $\omega \in \mathbb{R}^{\mathbb{M}}$ is also i.i.d Gaussian random variable with zero mean and variance $\sigma_{w}^{2}$.

e) The length $\mathrm{N}$ of $\mathrm{x}$ is sufficiently large i.e $\Phi^{T} \Phi=$ M. $I_{N}$.
Taking into consideration those terms, they derived the lower bound of the probability of error as follows;

$$
P_{e} \leq \frac{1}{\left[\frac{2 \pi P_{S I} \sigma_{w}^{2}}{\sigma_{w}^{2}+M P_{S I}}\right]^{N / 2}}
$$

\section{Proposed Optimal Solution}

In this section the integration of the prime work reviewed along with detailed description of the proposed scheme are discussed. As mentioned previously, singular base station with directive antennas, single relay equipped with multiple transceiver antennas with energy harvesting capabilities, and diverse types of end users are the three dominant components of the network considered. While the relay, in this model, is restricted to energy scavenged from the surrounding, both the base station and end users are battery powered. As shown in Fig. 2, we propose a two parallel phases protocol for the WPR system. For the total block duration $\mathrm{T}$ in Line 1 , we consider dividing it into, $\alpha \mathrm{T}$ and (1- $\alpha$ ) T, where $\alpha$ is the time-switching parameter. The relay harvests energy from the received signals from the $\mathrm{S}$ during $\alpha \mathrm{T}$, and then receives information from the same signal during $(1-\alpha) \mathrm{T}$. In the parallel phase,line 2, with constant time frame $\mathrm{T}$, the relay process and forwards the signal received from both nodes to the other end. As in Line 1 , the $\mathrm{S}$ and the $\mathrm{D}$ transmit the information signal to the relay are denoted as $x_{S}[k]$ and $x_{D}[k]$, respectively. In the following, we consider symbol-by-symbol transmission so that the time index k can be dropped and we simply write $x_{S}$ and $x_{D}$. The information signal received by the $\mathrm{R}$ after one block time $\mathrm{T}$ is given by;

$$
\mathbf{y}_{r}=\sqrt{P_{S I}} \mathbf{H}_{k, i} \mathbf{x}_{S}+\sqrt{P_{D I}} \mathbf{H}_{j, k} \mathbf{x}_{D}+\mathbf{n}_{r}
$$

Where $P_{S I}$ and $P_{D I}$ denote the source and end user information powers, $\mathbf{H}_{k, i}$ and $\mathbf{H}_{j, k}$ are the first-hop channel gains from the source to the relay and from the end user to the relay, respectively. The received signal at the relay will be corrupted by noise $\mathbf{n}_{r}$, which is modelled as an AWN random variable with zero mean and variance $\sigma_{r}^{2}$, denoted by $\mathbf{n}_{r} \sim\left(0, \sigma_{r}^{2}\right)$ The relay uses the received signal for energy harvesting during $\alpha$ $\mathrm{T}$ time and it ignores the negligible energy harvested from the receiver noise $\mathbf{n}_{r}$.

Hence, the harvested energy at the relay can be written as;

$$
E_{E H}=\alpha T \eta\left(P_{S E}\left|\mathbf{H}_{k, i}\right|^{2}\right)
$$

Where $P_{S E}$ is the power supplied by the source for energy harvesting and $0<\eta \leq 1$ denotes the energy harvesting efficiency. The EH receiver at the relay converts the RF received signal $E_{E H}$ to a direct current (DC) by a rectifier without the need to convert from RF band to baseband [10]. Thanks to the law of energy conservation, the harvested power at the relay (energy normalized by the symbol duration), denoted as, $Q_{r}$, is given by;

$$
Q_{r}=\zeta\left[\left\|h_{1} x_{s}\right\|^{2}\right]
$$

where $\zeta \epsilon[0,1]$ is the conversion efficiency.

That is said that, at that instance, the total power transmitted 
from the source and the end user for the relay operation during both lines at time $\mathrm{T}$ is;

$$
\begin{gathered}
P_{S}=\alpha T P_{S E}+(1-\alpha) T P_{S I} \quad \text { and } \\
P_{D}=T P_{D I}
\end{gathered}
$$

After $\mathrm{T}$ time, the relay now has enough energy storage for the consequent transmission of information.At this point, the first-hop received SNR at the relay can be expressed as;

$$
\gamma_{1}=\frac{P_{S I}\left|\mathbf{H}_{k, i}\right|^{2}}{\sigma_{r}^{2}} \text { and } \gamma_{2}=\frac{P_{D I}\left|\mathbf{H}_{j, k}\right|^{2}}{\sigma_{r}^{2}}
$$

For this case, information rate supplied by both source and destination at the relay during the $\mathrm{T}$ time can be expressed as,

$$
\begin{gathered}
R_{S}=(1-\alpha) T \log \left(1+\frac{\left|\mathbf{H}_{k, i}\right|^{2} P_{S I}}{\sigma_{r}^{2}}\right) \\
R_{D}=T \log \left(1+\frac{\left|\mathbf{H}_{j, k}\right|^{2} P_{D I}}{\sigma_{r}^{2}}\right)
\end{gathered}
$$

Assuming that channel matrices are i.i.d complex Gaussian random variables with zero mean and unit variance.

The evaluation of the proposed scheme concerning how much data can be compressed to compensate the data rate lost when allocating time for energy harvested, along with the upper bound of the probability of error after taking into consideration the prior mentioned work is simulated in the next section.

\section{Numerical Results}

In this simulation, it is assumed that the communication between the $\mathrm{S}$ and $\mathrm{EU}$ occurs through the relay or a direct link. To evaluate the performance of the proposed framework, a three consecutive timeslot scenario has been considered. During the first time slot, $\mathrm{S}$ is transmitting data to the relay. Following that, the relay receives energy from $S$. While receiving data from EU during both time slots. Lastly, the relay starts retransmitting the data to the other end during the last time slot.The work mainly focuses on assessing the performance boundaries of the wireless system considered, when performing CS to BPSK modulated signals from both $\mathrm{S}$ and $\mathrm{EU}$ nodes to the relay, whilst maintaining the data rate to be constant and meeting the power constraint.

To accommodate variable energy demand scenarios, reflected with different values of $\alpha$, met with appropriate range of compressibility alongside SWIPT have been considered.

Fig. 3 shows the target data rate achieved at the relay without SWIPT utilization and after applying TS SWIPT with different time allocation for $\mathrm{EH}$, and its relationship with source transmission power $P_{S I}$. The results show the shrinkage in data rate as the $\alpha$ increases, giving a frame of reference to the compression compensation required. Fig. 4

Fig.4 shows a plot of the lower bound of the probability of error for different $(1-\alpha)$ i.e time allocated to data transmission with different power transmitted by the source, providing a reference for field engineers depending on the grade of service they target. It is observed that, when the time allocated for data is at minimum of 0.3 and while the SNR is below 5

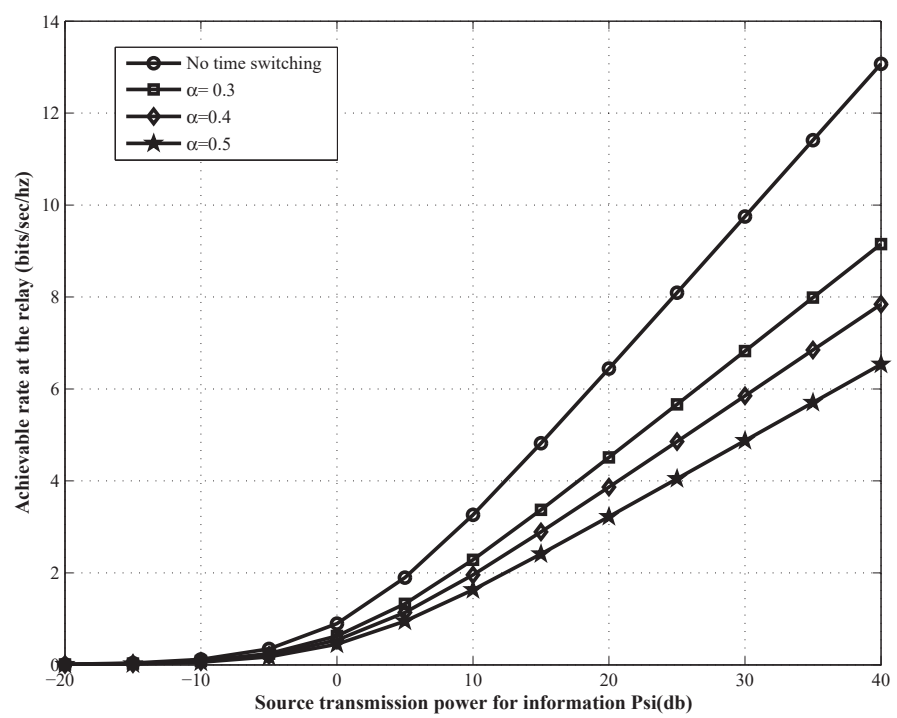

Fig. 3. Data rate achieved for the S-R link after T time with different time allocated for $\mathrm{EH}$

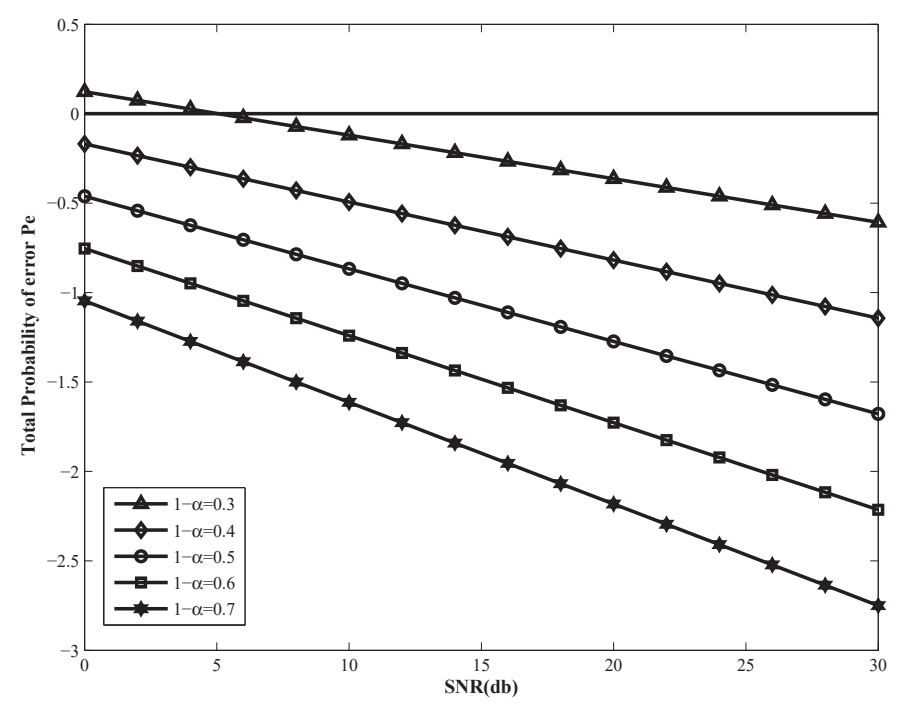

Fig. 4. Lower bound of $P_{e}$ of the proposed CS scheme with SNR values at the relay side

$\mathrm{db}$, the lower bound of probability of error is a positive value, proving that the sampling rate considered is aggressive making perfect reconstruction of the original data tough. As the time allocation for data transmission increases, meanwhile sampling rate $\mathrm{M} / \mathrm{N}$ rises, the lower bound of probability of error is a negative value, for the range of $0 \leq \mathrm{SNR} \leq 30$ making the reconstruction of the original information possible.

These results present some kind of reference of the probability of error of TS SWIPT system utilizing CS to maintain constant data rate while supplying energy.

\section{CONClusion}

The work proposed in this paper provides with consistency of supporting demanded data rate while employing simulta- 


$$
\begin{aligned}
& L(P, T)=\alpha \operatorname{T} \eta\left(P_{S E}\left|\mathbf{h}_{k, i}\right|^{2}+P_{D E}\left|\mathbf{h}_{j, k}\right|^{2}\right)+\beta_{1}\left((1-\alpha) T \log \left(1+\frac{\left|\mathbf{h}_{j, k}\right|^{2} P_{S I}}{\sigma_{r}^{2}}\right)-R_{T}\right)+\beta_{2}\left(T P_{S}-\alpha T P_{S E}-(1-\alpha) T P_{S I}\right)(23) \\
& \begin{aligned}
L(T) & =\left[T \eta\left(P_{S}^{*}\left|\mathbf{h}_{j, k}\right|^{2}\right)-(1-\alpha) T \eta\left(P_{S I}^{*}\left|\mathbf{h}_{j, k}\right|^{2}\right)\right]+\beta_{1}(1-\alpha) T \log \left(1+\frac{\left|\mathbf{h}_{j, k}\right|^{2} P_{S I}^{*}}{\sigma^{2}}\right)-\beta_{1} R_{I}+\beta_{2} P_{S}-\beta_{2} P_{S I}^{*}-\beta_{2}\left(P_{S}^{*}-P_{S I}^{*}\right) \\
& =(1-\alpha) T\left[\beta_{1} \log \left(1+\frac{\left|\mathbf{h}_{j, k}\right|^{2} P_{S I}^{*}}{\sigma^{2}}\right)-\eta\left(P_{S I}^{*}\left|\mathbf{h}_{j, k}\right|^{2}\right)\right]+T P_{S}^{*}\left[\eta\left|\mathbf{h}_{j, k}\right|^{2}-\beta_{2}\right]-\beta_{1} R_{I}+\beta_{2} P_{S} \\
& =F_{I}^{*}+T P_{S}^{*}\left[\eta\left|\mathbf{h}_{j, k}\right|^{2}-\beta_{2}\right]-\beta_{1} R_{I}+\beta_{2} P_{S}
\end{aligned}
\end{aligned}
$$

neous wireless information and power transfer scheme. This ensure the sustainable usage of SWIPT in remotely located active or passive sensing devices, which are an integral part of IoT. Although, time sharing of power and information has been considered along side compressive sensing in the current paper, employing network coding alongside the presented framework has a potential to explore.

\section{ACKNOWLEDGMENT}

The authors would like to thank Dr. Anas Amjad for his kind support and suggestion to formulate the system simulation platform.

\section{REFERENCES}

[1] L. R. Varshney, "Transporting information and energy simultaneously," in 2008 IEEE International Symposium on Information Theory. IEEE, 2008, pp. 1612-1616.

[2] P. Grover and A. Sahai, "Shannon meets tesla: Wireless information and power transfer." in ISIT, 2010, pp. 2363-2367.

[3] D. Mishra, S. De, and C.-F. Chiasserini, "Joint optimization schemes for cooperative wireless information and power transfer over rician channels," IEEE Transactions on Communications, vol. 64, no. 2, pp. 554-571, 2016.

[4] F. Benkhelifa, A. K. S. Salem, and M.-S. Alouini, "Simultaneous wireless information and power transfer for decode-and-forward mimo relay communication systems," in 2015 IEEE 81st Vehicular Technology Conference (VTC Spring). IEEE, 2015, pp. 1-5.

[5] C. Zhong, H. A. Suraweera, G. Zheng, I. Krikidis, and Z. Zhang, "Wireless information and power transfer with full duplex relaying," IEEE Transactions on Communications, vol. 62, no. 10, pp. 3447-3461, 2014.

[6] E. J. Candès and M. B. Wakin, "An introduction to compressive sampling," IEEE signal processing magazine, vol. 25, no. 2, pp. 2130, 2008.

[7] S. Joshi, K. Siddamal, and V. Saroja, "Performance analysis of compressive sensing reconstruction," in Electronics and Communication Systems (ICECS), 2015 2nd International Conference on. IEEE, 2015, pp. 724729.

[8] J. Le, J. C. Lui, and D.-M. Chiu, "On the performance bounds of practical wireless network coding," IEEE Transactions on Mobile Computing, vol. 9, no. 8, pp. 1134-1146, 2010.

[9] J. Chen and Q. Liang, "Theoretical performance limits for compressive sensing with random noise," in Global Communications Conference (GLOBECOM), 2013 IEEE. IEEE, 2013, pp. 3400-3405.

[10] X. Zhou, R. Zhang, and C. K. Ho, "Wireless information and power transfer: Architecture design and rate-energy tradeoff," IEEE Transactions on Communications, vol. 61, no. 11, pp. 4754-4767, 2013. 